\title{
Frontières
}

\section{Des cadavres apparents}

\section{Hans-Jürgen Greif}

Volume 23, numéro 1, automne 2010

Enquêtes sur le cadavre : 1. Fascination

URI : https://id.erudit.org/iderudit/1004027ar

DOI : https://doi.org/10.7202/1004027ar

Aller au sommaire du numéro

Éditeur(s)

Université du Québec à Montréal

ISSN

1916-0976 (numérique)

Découvrir la revue

Citer cet article

Greif, H.-J. (2010). Des cadavres apparents. Frontières, 23(1), 79-83.

https://doi.org/10.7202/1004027ar

Ce document est protégé par la loi sur le droit d'auteur. L'utilisation des services d'Érudit (y compris la reproduction) est assujettie à sa politique d'utilisation que vous pouvez consulter en ligne.

https://apropos.erudit.org/fr/usagers/politique-dutilisation/
Cet article est diffusé et préservé par Érudit.

Érudit est un consortium interuniversitaire sans but lucratif composé de l’Université de Montréal, l'Université Laval et l'Université du Québec à Montréal. Il a pour mission la promotion et la valorisation de la recherche. https://www.erudit.org/fr/ 


$\begin{array}{lllllll}\text { R } & \text { E } & \text { G } & \text { A } & \text { R } & \text { D } & \text { S }\end{array}$

\section{DES CADAVRES APPARENTS}

\author{
Hans-Jürgen Greif, Ph.D., \\ professeur associé, Département des littératures, Université Laval.
}

Dans son tout nouveau livre, Histoires de jardin, un ravissant recueil d'essais sur les affres, les peines et les joies que peuvent nous offrir les jardins, Eva Demski raconte que, lors d'un voyage à Paris, elle n'avait pas remarqué l'indication routière pour Verdun.

Je n'avais jamais encore rencontré une terre aussi sombre, aussi hostile que celle-ci. Les massacres remontaient à plusieurs décennies, mais la terre semblait ne pas s'être remise de ce qu'elle avait dû absorber. On pourrait croire qu'une végétation particulièrement luxuriante envahit les champs de la mort. Ici, ce n'était pas le cas. [...] La terre trop nourrie des vrais cimetières est plus aimable; elle donne ce qu'elle peut aux petits quadrilatères qui les constituent. Dans un cimetière de mon enfance se trouvaient des pommiers particulièrement beaux. C'était faire preuve d'audace d'en manger les fruits. Nous les appelions les pommes des cadavres. [...] Peut-être n'aimons-nous guère contempler la terre à cause de son lien avec la mort (Demski, 2009, p. 27 ; je traduis).

Ces pommes de cadavres - Leichenäpfel en allemand - rappellent celle préparée par la marâtre de Blanche-Neige : un fruit beau, appétissant, dont la moitié est empoisonnée. La méchante reine, déguisée en vieille femme, veut assassiner sa rivale, «mille fois plus belle qu'elle», lui avait dit le miroir. Pour convaincre sa victime que rien n'est plus inoffensif qu'une pomme, elle en mange la moitié. On connaît le reste du conte des frères Grimm : la jeune fille n'est morte qu'en apparence, le morceau de pomme qu'elle s'apprêtait à avaler lui est resté dans la gorge. Un beau prince est de passage. Quand il voit la «morte», il en tombe amoureux. Les sept nains lui cèdent le cercueil. Mais lors du transport, les porteurs trébuchent. Le morceau de pomme sort de la gorge, Blanche Neige revient à la vie, elle se lève et épouse le prince. La marâtre est invitée à la fête où on la fait danser dans des pantoufles en fer, chauffées à blanc. Elle est condamnée à danser jusqu'à ce que mort s'ensuive. La mort n'aura été qu'un jeu, comme dans La Belle au bois dormant: les jeunes filles des deux contes sont à l'image de ces saintes dont le cadavre ne se décompose pas mais affiche tous les signes de la vie: joues roses, corps intact. Le cadavre peut revenir parmi les vivants, nouveau Lazare - mais cette fois, qu'on se le dise, cette revenante, ce revenant de l'au-delà, nous racontera en détail son voyage. C'est l'un des rêves les plus puissants de l'être humain.

Le terme allemand Leiche a des connotations plus complexes que son équivalent français, alors que Kadaver s'emploie avant tout pour un animal mort. Leiche ne désigne pas seulement le corps mort, il va plus loin en impliquant que le cadavre est déjà en train de se décomposer. Il contient du poison, il est dangereux. En Europe, dans les campagnes, jusque dans les années 1950, il fallait honorer le mort en lui offrant une dernière fête (le Leichenschmaus) où l'on mangeait, buvait, racontait des anecdotes drôles et des épisodes moins réjouissants de la vie du défunt. Mais ensuite, il fallait se débarrasser de cette présence menaçante en l'éloignant, en l'ensevelissant six pieds sous terre. Dans son essai Hitler d'après Speer, Elias Canetti a souligné, à juste titre, la position des cadavres, une fois enterrés: ils sont couchés, alors que nous, les vivants, sommes debout devant leur sépulture (Canetti, 1972, p. 7-39). Les stèles, les monuments sont habituellement debout, chez les juifs tout comme chez les chrétiens, les catholiques surtout (depuis l'incinération quasi systématique des cadavres en Occident, cette tradition est en 
C'EST DE CELA QUE PARLENT DEUX PUBLICATIONS

RÉCENTES, LE LIÈVRE DE PATAGONIE, DE CLAUDE LANZMANN, ET ZONE, DE MATHIAS ÉNARD'. SEULEMENT, DANS CES DEUX LIVRES, LES CADAVRES

DISPARAISSENT, DEVIENNENT INVISIBLES, SE VOLATILISENT, SE TRANSFORMENT EN FUMÉE, OU ILS SE DÉROBENT, SONT PROFANÉS, RIDICULISÉS, SERVENT POUR ACCOMPLIR DES ACTES HAINEUX.

train de se perdre, sauf chez les juifs). Les plantes ornementales sur une tombe poussent vers le ciel, à la verticale, elles se dressent comme nous vers la lumière. Elles fleurissent à la manière de leurs congénères; cependant, leur odeur est différente - les racines se nourrissent du cadavre, elles confèrent par le fait même un caractère particulier aux fruits des arbres poussant dans l'enceinte d'un cimetière. Ces fruits sont potentiellement dangereux pour la santé puisque leur suc provient du poison du mort. D'où le geste audacieux dont nous parle Eva Demski.

C'est un truisme de dire que le cadavre est l'enveloppe de celui que nous avons connu vivant. Cet objet froid et durci, déjà rongé par les forces destructrices de la Nature, est tout ce que la Mort nous a laissé. Le combat entre le vivant et sa dernière adversaire a été, peut-être, long et héroïque, ou bref et violent. Il reste que personne n'en sort vainqueur. D'habitude, la Mort s'annonce par les maladies qui sont ses messagères. Le vivant se prépare, il sait ce qui l'attend, il a vu des cadavres. Alors il met de l'ordre dans ce qui restera de lui sur cette terre, il distribue ce qu'il a accumulé pendant sa vie. Ceux qui lui survivent savent que, tôt ou tard, ils le suivront. Ils répètent les mêmes gestes, avant de rejoindre la file de ceux qui les ont précédés et qui se perdent dans l'infini. Ils espèrent que les maladies ne les feront pas souffrir trop longtemps, qu'un remède, un coup de baguette magique leur permettra de ruser pendant un temps, tout comme dans d'autres textes des frères Grimm, et qu'en fin de compte, ils rencontreront la mort dignement et en connaissance de cause (connaissance qui ne peut être et ne sera jamais complète, la médecine n'étant pas une science exacte).

Mais qu'arrive-t-il si la mort nous surprend à l'improviste? Si elle nous met le couteau sous la gorge et la tranche, sans nous avertir? Nous transforme en chose qui ne signifie plus rien, destinée à disparaître et à fertiliser le sol qui nous couvre? C'est de cela que parlent deux publications récentes, Le lièvre de Patagonie, de Claude Lanzmann, et Zone, de Mathias Énard ${ }^{1}$. Seulement, dans ces deux livres, les cadavres disparaissent, deviennent invisibles, se volatilisent, se transforment en fumée, ou ils se dérobent, sont profanés, ridiculisés, servent pour accomplir des actes haineux: on se venge alors sur la dépouille parce qu'on n'a pas pu mettre la main sur l'individu quand il était encore en vie. Pourquoi les hommes font-ils cela? Peut-on s'amuser avec un cadavre? En rire?

L'ouverture du Lièvre de Patagonie occupe tout le premier chapitre qui nous paraît très long, tant les horreurs évoquées par l'enfant Claude Lanzmann nous jettent à terre. Il parle de sa fascination pour la guillotine, instrument inventé par le docteur Joseph-Ignace Guillotin (le terme date officiellement de 1790), mais surtout de ce qui se passe dans les yeux du condamné, audelà même de sa mort. Le petit Claude en fait des cauchemars :
«On me débitait en tranches, plates comme des planches, d'une épaule à l'autre, en passant par le sommet du crâne» (p. 16). Il relate la décapitation publique d'un assassin en série allemand, Eugen Weidmann, survenue un an avant la Seconde Guerre, une mise à mort fortement médiatisée, avec des photos célèbres que Lanzmann scrutera plus tard, quand il aura vu les milliers de documents menant à la réalisation de la série Shoah. Weidmann, Lanzmann: les noms sont trop proches pour ne pas le frapper, lui ainsi que son entourage, et faire croire au garçon de treize ans qu'un funeste destin l'attend. L'exécution de l'Allemand sera la dernière à l'extérieur d'une cour de prison (en France, la peine capitale ne sera abolie qu'en 1981, sous François Mitterrand). Au moment où il rédige ses mémoires, Lanzmann craint toujours le retour de la barbarie, «il suffit d'un changement de majorité, d'un vote, d'une grande peur» (p. 16). Ce sont les regards des condamnés qui hantent l'auteur; ceux de Sophie et Hans Scholl, ceux des conjurés du 20 juillet qui ont tous péri, soit sous la hache, soit sous le lourd couteau de la guillotine allemande qui peut tenir dans une pièce à plafond bas. Il y a les derniers regards des officiers marocains accusés d'avoir fomenté un complot contre Hassan II. Le monde entier les voyait puisque Paris Match avait publié leurs photos: «[L]'ultime regard d[u général] Medbou, éperdu d'effroi et d'incrédulité, inspire la plus grande pitié» (p. 21). Il y a les visages de jeunes Chinois, terrifiés, hurlant leur douleur et le refus avant qu'une main ne courbe leur tête vers la terre, et pour dégager la nuque et pour forcer la personne (qui n'en est déjà plus une) à mourir dans une posture de pénitente: les photos, les films - l'exécution en Chine est toujours publique - montrent le tir et le corps qui s'effondre lentement, «dérisoire amas de chiffons » (p. 22). Ou les images prises par l'armée impériale japonaise, en 1937, à Nankin, où trois cent mille civils et soldats furent massacrés de toutes les façons possibles. L'Homme a l'imagination fertile pour expédier son prochain dans les jardins élyséens, lui faisant payer cher le passage : il faut le matraquer, le battre, le torturer avant de le libérer pour l'éternité.

La documentation sur la cruauté humaine est écrasante. À Canberra, l'horreur ultime attend Lanzmann dans le musée de la guerre avec deux photos sur lesquelles on voit le sabreur et sa victime, un grand Australien au long cou. Dans une lettre à ses proches, le bourreau se vante de la précision du geste, jusqu'aux derniers détails; c'est un précis de l'art de la décapitation. Ailleurs, dans une salle du Prado, le cinéaste contemple et revisite le célèbre Fusilamientos del 3 de Mayo de Goya, où les condamnés se jettent devant les balles des fusilleurs. Goya, dont les gravures décrivant les horreurs de la guerre restent des documents qui ne seront jamais assez cités et montrés ${ }^{2}$. Aussi horrifiant que cela puisse paraître, il ne faut pas fermer les yeux devant le garrottage, spécialité espagnole (abolie en 1978), pas plus que devant les vidéos amateurs d'exécutions d'otages et de «traîtres », par des intégristes islamiques. Ce que les médias nous cachent, ce sont des scènes d'une sauvagerie et d'une barbarie sans nom. Jugée trop violente pour nos yeux d'Occidentaux si peu habitués à la cruauté physique, nous ne voyons jamais la partie principale de la scène, rien que le début, où la victime, agenouillée, est pétrifiée de peur, anesthésiée par ce qui va suivre, car elle sait ce qui se passera: les imprécations du «juge», la condamnation à mort, le «juge» qui sort son coutelas, un couteau de boucher, en criant Allahu akbar.

Mais un cou humain, même amaigri, n'est pas seulement fait de chair tendre: il y a des cartilages et les vertèbres cervicales. Le tueur se sert alors [du couteau] à la façon d'une scie [...] dans des jets et des éclaboussures de sang, insoutenable va-et-vient qui nous fait vivre jusqu'au bout l'égorgement d'un homme comme celui d'un animal, porc ou mouton (p. 29). 
Censurer ces vidéos au nom de la déontologie est inacceptable: si nous regardions ces boucheries en Irak, en Afghanistan, en Arabie saoudite et ailleurs dans le monde musulman, notre vision de l'Islam et de sa loi ne serait plus jamais la même. Mais nous détournons le regard. Rares sont ceux qui soutiendraient de telles images.

Sommes-nous plus humains, plus civilisés en Amérique que ces barbares? La peine de mort se retrouve partout dans le monde. Les Américains grillent les condamnés sur une chaise électrique, ils préparent une chambre à gaz, ils pendent, injectent des poisons puissants, tout cela au nom de la loi. Des gens assistent à l'exécution; plus tard, ils attestent que tout s'est déroulé selon les règles.

« Nul signe de peur, mais tout entier peur, rigidifié par la peur» (p. 28), voilà ce qui caractérise le visage du condamné: derrière cette liste d'horreurs devant public, commises par quelque mutant de l'espèce humaine, liste pour laquelle nous manquent les qualificatifs, liste non exhaustive, s'en dessine une autre, muette. Plus de six millions de juifs, dont la moitié sont des Polonais, des milliers de gitans, d'homosexuels, de prisonniers politiques, tous assassinés froidement et sauvagement par les nazis, d'abord torturés, utilisés comme cobayes, puis gazés, pendus, décapités. Les survivants se souviennent, ils parlent. Mais ils ne disent pas, ne peuvent pas dire ce qui est arrivé une fois les portes des camions, des chambres à gaz closes derrière les victimes, jusqu'à trois mille à la fois. Il n'y a jamais eu de caméras qui ont filmé, dans le noir, à l'infrarouge, le grouillement de ces milliers de corps nus qui tentent d'échapper au gaz qui étouffe, brûle les poumons. Les bourreaux n'ont pas installé des micros pour capter les cris, les hurlements de peur, de rage pour survivre, cette dernière lutte pour une bouffée d'air non empoisonné.

Toujours, en ouvrant les lourdes portes en métal, les meurtriers découvraient une pyramide de corps humains où le plus fort se trouvait tout en haut après avoir escaladé cette tour mouvante de chairs mortes et flasques.

C'est à cela qu'est consacrée la dernière partie du livre de Lanzmann: dire l'indicible, montrer ce qui n'existe plus, faire revivre ce qui est mort. Plus de six millions de cadavres. Plus de six millions d'hommes, de femmes, d'enfants vivants, menant chacun leur vie, avant la décision de l'infernal triumvirat Hitler, Goebbels, Göring, de les exterminer, terme utilisé pour anéantir la vermine. Les juifs sont de la vermine qu'il faut ausrotten, vernichten. Dans une maison infestée, les exterminateurs viennent le matin; ils vaporisent le poison. Les occupants ne peuvent pas retourner chez eux avant un temps. Les fenêtres, les portes sont closes. Les exterminateurs savent combien de temps doit s'écouler pour tuer ce qu'ils visent: rats, souris, termites, fourmis, poux, puces, punaises, etc. Quand c'est terminé, on revient, on nettoie, on jette. Les membres du triumvirat et ses aides ont tout calculé. Ils compteront chaque sujet exterminé. Les registres sont là. Les noms y sont soigneusement inscrits, avec la date d'arrivée et celle de la mort. Ce qui manque: le passage de la vie à la mort. Il y a eu des témoins, allemands, mais surtout polonais, ukrainiens, lettons. Par leur bouche, les cadavres recommencent à parler. Des villageois pour la plupart, de Prostyn, Poniatowo, WolkaOkraglik, à côté d'un autre village, Treblinka, où, de juillet 1942 à août 1943, six cent mille juifs ont été assassinés.

Ils parlent, se rappellent des convois, des visages de ceux qui sont sortis des wagons, la sélection rapide, d'un côté les malades, les faibles, les vieux, les enfants qui sont immédiatement envoyés à la chambre à gaz. Ils sont morts à peine deux heures plus tard. Leurs cadavres seront brûlés ou, si les fours ne fournissent pas, jetés dans des fosses, arrosés de gazoline, mais le feu ne les consume pas complètement. Quelques jours plus tard, la terre bouge, ondule, les cadavres en décomposition rappellent aux vivants leur présence, tant aux bourreaux qu'à ceux qui vont les suivre quand ils seront trop malades et trop faibles pour servir. Ce sera bientôt. D'autres témoignages vont se joindre aux leurs, de Sobibor, Belzec, Chelmno, Oswiecim (Auschwitz). La gravité de ce qu'ils disent varie, mais le résultat est toujours le même: des cadavres, des montagnes de cadavres.

Un homme raconte que, pour tuer le temps, lui et d'autres exterminateurs, jouaient aux cartes. Les mises étaient élevées. Une nuit, un de ces hommes a payé sa dette en jetant sur la table du débiteur un objet enveloppé dans du papier journal. C'était une mâchoire couverte de sang frais, avec plusieurs dents en or (p. 498).

[S]i j'avais trouvé un hypothétique film muet de quelques minutes, tourné en secret par un SS et montrant la mort de trois mille personnes dans une chambre à gaz, je ne l'aurais non seulement pas intégré à mon film, mais je l'aurais détruit. [...] Je n'ai pas réalisé Shoah pour répondre aux révisionnistes ou négationnistes: on ne discute pas avec ces gens-là, je n'ai jamais envisagé de le faire. Un chœur immense de voix dans mon film - juives, polonaises, allemandes - témoigne, dans une véritable construction de la mémoire, de ce qui a été perpétré (p. 488).

Faire parler les cadavres. Le but de Lanzmann : les rassembler, un à un, ne plus considérer la masse de ces six millions comme un ensemble anonyme, mais faire en sorte que ceux qui regardent son film se rendent compte de l'individualisation de cette population, plus importante que celle de la Suisse, de la Norvège ou de la Suède. Ces juifs venus de partout du continent, Pologne, Allemagne, Autriche, France, Italie, Hongrie, Grèce, des Balkans, après de longs voyages, forment une tour de Babel qui se tait au moment où s'ouvrent les portes derrière lesquelles ils rencontrent leur mort, chacun, côtoyant d'autres, mais chacun seul. Il y a un palimpseste qui parcourt toute la narration de Shoah, celui des survivants. Pas seulement ceux que les nazis n'ont pas eu le temps de tuer, mais ceux que le sort a fait échouer ailleurs, en Amérique, en Afrique, en URSS. Nous savons que des familles entières ont péri dans les camps. Mais beaucoup d'autres victimes gardaient le contact avec des proches sur tous les continents. Ces survivants n'ont jamais pu faire le deuil des disparus. Ils ont connu le sentiment de la perte; pour la forme, ils ont récité le kaddish, la prière pour commémorer ces morts dont ils n'ont pas inhumé le cadavre. Car sans l'enveloppe corporelle, le frère, la sœur, le père, la mère ne sont pas vraiment morts, même si l'on peut consulter les registres de la SS, trouver noms, dates de naissance, dates de décès. C'est le corps qui manque.

Ce sont des cadavres apparents qui hantent les survivants, des photos de famille où les visages des disparus ont été découpés. Au moins, il faut faire un voyage en Pologne. Ce village, Treblinka, existe-t-il ? Lanzmann écrit: "J'avais beau n'avoir jamais douté de l'existence de Treblinka, la malédiction pour moi attachée à ce nom portait en même temps sur lui un interdit absolu, d'ordre quasi ontologique, et je m'apercevais que je l'avais relégué sur le versant du mythe ou de la légende» (p. 491). Et de continuer, après avoir lu TREBLINKA en arrivant à la gare: "Treblinka devint vrai, le passage du mythe au réel l'opéra en un fulgurant éclair, la rencontre d'un nom et d'un lieu fit de mon savoir table rase, me contraignant à tout reprendre à zéro» (p. 492). C'est la lecture de ce toponyme terrible qui évoque tant d'existences transformées en fumée, la puanteur qu'il contient (car les villageois sont submergés par l'insoutenable odeur qui se dégage des cheminées des fours crématoires), ce nom de Treblinka est un ordre: assez lu, assez écrit, assez discuté. Maintenant, il faut passer à l'acte, créer un monument-document qui durera jusqu'à la fin des temps. La banderole sous l'enseigne à la gare dit: "Plus jamais », seule allusion à l'histoire de cette bourgade. 
Il faut aller beaucoup plus loin et faire comprendre ce que dit un «héros » sorti vivant du ghetto de Varsovie, Antek: «Claude, si vous pouviez lécher mon cœur, vous seriez empoisonné » (p. 501). Il a vécu une existence antérieure à celle qu'il mène dans ce kibboutz en Galilée, une vie si effroyable que les bureaucrates israéliens refusent de le laisser parler pendant le tournage de Shoah. Mais ce poison, tous les survivants le portent en eux. Pour le neutraliser, Lanzmann montre ce qui reste des traces juives à Auschwitz, ville à quatre-vingts pour cent juive avant la guerre: les stèles de l'ancien cimetière et leurs caractères hébraïques. Ici, sous la terre, reposent encore les restes de ceux qui avaient eu la chance de mourir avant la Shoah (depuis l'œuvre de Lanzmann, le terme a souvent remplacé celui d' "Holocauste», signifiant «sacrifice par le feu», alors que Shoah porte le sens de «catastrophe, destruction, anéantissement»). En montrant ces images, les survivants voient des objets reliés à leur culture au lieu de lire des lettres qui n'établissent aucun rapport avec les morts. Une femme confirme: «Ici, il y avait toutes sortes de gens, de tous les côtés du monde, qui sont venus ici, qui ont été dirigés ici... Tous les juifs sont venus ici. Pour mourir» (p. 510). Avec ce seul témoignage de cette Polonaise, madame Pietyra, qui a tout su, qui a vu, les survivants peuvent commencer leur travail de deuil : maintenant ils savent que la catastrophe a vraiment eu lieu et que l'anéantissement n'est pas une fable, quoi qu'en disent les négationnistes.

Quelque temps après avoir vu l'œuvre monumentale de Lanzmann, j'ai été invité à prononcer à Berlin une conférence sur la mémoire dans les œuvres de ce que l'on appelle encore les «écrivains migrants». Un matin, j'ai traversé un marché à ciel ouvert; c'était une place où l'on vend, deux fois par semaine, des légumes, des fruits, des fleurs, des victuailles de toutes sortes. Je ne savais pas qu'à Berlin, il existe à côté de l'œuvre architecturale bouleversante de Libeskind et de plusieurs autres musées en l'honneur des juifs assassinés, un monument qui n'en est pas un, tellement il ne paie pas de mine. Il n'est pratiquement jamais mentionné. En face de la mairie de Steglitz, avec Dahlem une des parties de la ville où s'étend l'Université libre (Freie Universität), se dressent quelques grandes plaques de verre dans lesquelles sont gravés à l'acide les noms de centaines d'habitants juifs disparus de cette partie de la ville. Les vitres sont transparentes. J'ai parcouru les listes, enregistré les dates de naissance et de mort, invariablement survenue entre 1941 et 1945. Les listes sont longues. J'ai rapidement perdu intérêt à lire les noms de gens que je ne connaissais pas. Mais tout à coup, je me suis rendu compte que ce n'était pas qu'une simple liste de noms, mais que ces noms, qui ne me parlaient que par leur consonances, indiquant souvent qu'il s'agissait de personnes d'origine ashkénaze, se faisaient entendre d'une manière on ne peut plus éloquente. M'arrêtant à un de ces noms, à peu près à la hauteur de mes yeux, je me suis vu, moi, le vivant, dans le reflet de la vitre. Le coup a été dur à encaisser. Quel mérite avais-je donc eu pour ne pas faire partie de cette liste? Aucun: l'année de ma naissance, la machine d'extermination avait déjà atteint sa vitesse maximale, j'étais trop jeune pour en connaître l'existence. Mais je descendais de ceux qui avaient conçu cette barbarie, j'en faisais partie. J'étais là, bouleversé par ce que voyais: les cadavres derrière ces noms se redressaient, s'habillaient, repartaient de Pologne pour réintégrer leur place à côté de moi, dans ce quartier berlinois d'où ils avaient été déportés. Je les entendais, ils m’interrogeaient. Je leur dois encore des réponses.

Le cadavre apparent, celui qui semble être mort mais continue à poser des questions qui resteront à jamais suspendues en l'air, surgit au fil d'une des plus longues phrases itératives de la littérature contemporaine. Il s'agit du roman de Mathias Énard, Zone. Le narrateur, Francis Sirvain Mirkovic, fils d'une mère croate et d'un père français, effectue un voyage de nuit, très semblable à celui de La Modification de Michel Butor, de Milan à Rome. Mirkovic a participé aux combats fratricides dans l'ancienne Yougoslavie. Il a assisté aux atrocités commises envers la population civile serbe avant de s'enrôler dans les services secrets français. Il a effectué de nombreux voyages dans sa "Zone», le Maghreb, le Proche et le Moyen-Orient. Sa mallette, attachée au porte-bagages, est destinée à un haut dignitaire du Vatican. Elle contient des listes avec des milliers de noms, des photos prises dans des camps de transit, de concentration, d'extermination. C'est une gigantesque entreprise de documentation sur les atrocités des guerres qui se sont déroulées autour du bassin de la Méditerranée. Juifs, Serbes, Croates, Bosniaques, Turcs, Algériens, Espagnols, Italiens, Grecs, Libanais, des hommes, des femmes, des enfants, déportés, torturés, battus à mort, éventrés, décapités, pendus, gazés. Il a enregistré des témoignages, connaît les noms, les lieux des crimes. Des bourreaux, il a les adresses et leurs procédés favoris lors de la mise à mort de prisonniers. Avec l'argent qu'il recevra du Vatican, il tentera - rien n'est plus sûr - de changer de vie.

Ce bref résumé donne une fausse image de l'œuvre. C'est comme si l'on essayait de réduire Shoah à quelques séquences (ce qui a été fait, en Pologne, où la série a été vilipendée comme un déshonneur national). L'énorme masse d'informations amassées par Énard peut surprendre. Le lecteur est pris dans le maelström de la pensée du narrateur pour être aspiré jusqu'au fond de la barbarie la plus innommable du monde (est-elle encore possible, après les crimes nazis?). Si le lecteur est ballotté d'un coin du monde méditerranéen à l'autre, d'une histoire d'amour avorté à l'autre, d'une mort à l'autre, d'une guerre à l'autre, il en sort aussi épuisé et bouleversé qu'après le visionnement de Shoah. Les deux œuvres sont concomitantes et non pas parallèles, car elles se touchent en plusieurs points, dont les moindres sont la cruauté de l'humain et les assassinats des juifs que l'occupant allemand a, bureaucratie et méthode obligent, envoyés dans des wagons destinés à transporter du bétail, vers les camps d'extermination. Contrairement aux cadavres invisibles de Lanzmann, ceux de l'immense monologue intérieur de Mirkovic sont déposés devant nos yeux. Ils accusent le monde entier de ne pas avoir voulu voir leur souffrance et celle de leur pays, selon la vieille formule de la guerre de Troie: "Si tu me tues, un des miens te tuera. » Nous ne sommes plus dans la loi du Livre juif, où le tort commis est réparé par une punition. Comme pour les juifs assassinés en Pologne, il n'y a jamais eu de tort, sauf celui d'être né juif, serbe, croate, slovène, hongrois, grec.

Le commando de Mirkovic s'arrête par hasard (mais il n'y a pas de hasard, ni dans la vraie vie ni dans les romans) devant une maison croate. Un vieillard et son chien ont été abattus sur le seuil. Les hommes entrent. Une vieille femme est étendue sur le lit conjugal, des cierges sont allumés. «[E]lle n'a rien entendu, sur son lit de mort, ni les rafales dehors, ni les pas dans sa maison, ni les rires de ceux qui ont planté, bien droit, ce grand crucifix au milieu de son ventre, dont l'ombre absurde danse sur le mur [...] ». Devant ce cadavre mutilé et déshonoré qui crie vengeance, l'ami de Mirkovic

pleure comme une fontaine silencieuse sans se cacher, pourquoi, les cadavres il en a vu des quantités, des jeunes, des vieux, des mâles, des femelles, des brûlés noircis, des découpés, des mitraillés, nus, habillés ou même déshabillés par une explosion, pourquoi celui-ci, Andrija mourra quelques semaines plus tard, il aura le temps de venger ses propres larmes, de cautériser ses pleurs dans les flammes, de ravager à son tour des corps ennemis, des maisons, des familles [...] Andrija le furieux il vengeait cette grand-mère inconnue dont il ne m'a jamais reparlé, j'ai encore dans l'œil 
l'ombre du Christ sur la tapisserie à fleurs, rien n'avait été dérangé, aucune inscription vengeresse sur les murs, rien, c'était un étrange miracle ce crucifix fiché Dieu sait comment dans la chair de cette vieille femme [...] (p. 160-161).

Des récits de scènes comme celle-ci, le livre en donne des douzaines; elles sont à la limite du supportable. Pour une fois, rien ne nous est épargné. Nous lisons; le film se déroule dans notre tête; nous gardons en mémoire ces mises à mort atroces, au lieu de ces «capsules» infâmes montrées au téléjournal, coupées «pudiquement», ces capsules dénoncées à juste titre par Lanzmann, car l'être humain occidental a «la sensibilité trop fragile», alors que ces actes sont commis devant la porte du voisin et dans sa maison. Nous entendons les râles, les cris des hommes, des femmes, des enfants torturés, violés, assassinés. Il est plus commode de regarder ailleurs, de se réfugier dans son quant-à-soi, de se boucher les oreilles, ni vu ni connu. «Laissezles s'entretuer, jusqu'au dernier, m'a dit une amie très âgée qui a vécu toutes les guerres du $\mathrm{XX}^{\mathrm{e}}$ siècle. L'Homme sera toujours le loup de l'Homme, rien ne changera, Pline et Hobbes avaient raison.» Et de conclure: "Bon débarras».

C'est une question à première vue étrange qui se pose devant ces deux œuvres, et de beaucoup d'autres qui les rejoignent, celles des Semprun, Primo Levi, Antelme, Rousset, Böll, Grass, Malaparte, Philip Roth, Canetti, Joseph Boyden, la liste est longue. Sommes-nous, comme Lanzmann semble le suggérer, devant des mutants de l'espèce humaine, en train de prendre le contrôle du Mal, où la mort est ritualisée dans ses formes les plus abominables? Il n'y a pas de réponse à la barbarie, il ne reste que la tristesse profonde et la piété. Dans un roman dont Mirkovic lit des bouts qui épousent les méandres de sa pensée, surgit, au détour de l'insurrection palestinienne, une combattante dont le mari a été fauché par des mitrailleurs israéliens. Des amis lui ont amené le corps. Elle se doit de le laver et de le préparer pour l'enterrement:

À genoux auprès de Marwan, c'est bien lui, tout d'un coup. Elle le voit là malgré la distance de la mort. Il est revenu dans son corps. [...] Son torse. [...] Odeur de viande, de mort. [...] Elle prend une éponge, et renverse le contenu d'une bouteille d'eau sur Marwan. Intissar ne tremble plus. Elle ne pleure plus. Elle le caresse doucement. Elle efface petit à petit les traces de sang coagulé sur le torse, autour de la bouche, du nez, sur le ventre, délicatement. Marwan le guerrier (p. 311-312).

C'est Antigone, c'est Priam qui redonnent la dignité au corps des morts qui redeviennent, pour un temps trop bref, ce qu'ils ont été autrefois. Tout au long du roman, le point de référence d'Énard est la guerre de Troie, où celle des hommes se reflète, parfois de façon caricaturale, dans celle que mènent les dieux, à la différence que les dieux sont immortels ${ }^{3}$. Les guerriers d'antan en ont engendré d'autres, les veuves d'Ajax, d'Hector, de tant d'autres qui sont «tombés pour la gloire» dans cette boucherie, ces veuves pleurent leurs morts, à Troie comme à Belgrade, ou Alger, Tripoli, Tel Aviv, Istanbul, «Beyrouth et Barcelone se touchent par pliage sur l'axe Rome/Berlin» (p. 286).

Ce roman est un livre sur et contre la haine. Écoutons enfin un souvenir de Mirkovic qui évoque un ancien ami parisien, devenu fou:

Yvan détestait l'Église catholique qu'il fallait mettre au pas et haïssait avec une belle furie tout ce qui n'était pas lui, les juifs les communistes les Arabes les Britanniques invertis les Jaunes grouillants les capitalistes pervers les politiciens corrompus une liste interminable de haines et de dégoûts motivée par la lecture d'opuscules paranoïaques et hallucinés ornés de croix gammées, de croix pattées, de rose-croix, de toutes les croix possibles et imaginables sauf la croix de Lorraine, [...] Yvan avait une vraie passion, ardente et contagieuse, je me suis laissé convaincre par son admirable rage, sans doute avais-je des prédispositions [...] (p. 447-448).

Voici au moins une réponse partielle à la question que nous n'arrêtons pas de nous poser: pourquoi ces massacres interminables? Nous savons depuis Caton l'Ancien ("Delenda est Carthago ») que, si l'on répète inlassablement la même chose, la même conviction, on finira bien par convaincre les autres que l'on dit vrai. Des figures rhétoriques bien employées, le but du discours clairement identifié et les répétitions en boucle : le résultat sera la haine de l'autre qu'il faut assouvir par tous les moyens. Dans Mein Kampf, Hitler a comparé le juif à un asticot (Made en allemand) vivant dans et se nourrissant du corps du peuple allemand. On n'a qu'à entailler ce corps endolori au bon endroit, c'est la tête d'un juif que porte cet asticot parasite qui en émerge.

N'oublions pas le passé, jamais, mais apprenons à pardonner. Les «héros » sont rares. Ils sont souvent des fabrications après les événements. L'effet cathartique des œuvres de Lanzmann et d'Énard est immédiat: personne ne peut rester indifférent puisqu'elles montrent au grand jour la folie de l'être humain, si différent de l'image que nous lui prêtons, ses rages meurtrières, les souffrances, les humiliations, la terreur infligées à l'autre parce qu'il appartient à une ethnie, à une religion différentes. Les deux auteurs mettent en marche un énorme rouage devant nos yeux. Rien n'en arrête le mouvement implacable, à la logique incompréhensible, comparable à celle de la machine qui se détraque dans «La colonie pénitentiaire» de Kafka. Ce qui reste après ces œuvres: le goût du sang dans la bouche, l'odeur de la chair brûlée, des images dont la beauté est aussi terrifiante que leur laideur.

\section{Bibliographie}

BARICCO, A. (2006). Homère, Iliade, Paris, Albin Michel, coll. «Les Grandes Traductions », $179 \mathrm{p}$.

CANETTI, E. (1972). "Hitler nach Speer», dans Die gespaltene Zukunft, Munich, Carl Hanser Verlag, 140 p.

DEMSKI, E. (2009). Gartengeschichten (Histoires de jardins), illustrations de Michael Sowa, Francfort-sur-le-Main, Insel, 237 p.

ÉNARD, M. (2008). Zone, Arles, Actes Sud, 521 p.

LANZMANN, C. (2009). Le lièvre de Patagonie. Mémoires, Paris, Gallimard, $558 \mathrm{p}$.

PÉREZ-REVERTE, A. (2008). Un jour de colère, trad. de l'espagnol par François MASPERO, Paris, Le Seuil, 354 p.

\section{Notes}

1. Claude Lanzmann (2009) et Mathias Énard (2008); afin d'éviter d'alourdir le texte, les chiffres entre parenthèses renvoient aux livres en question.

2. C'est le livre d'Arturo Pérez-Reverte, Un jour de colère (2008), qui suit, littéralement, minute par minute, les événements du 3 mai 1808. Certains quartiers madrilènes s'étaient rebellés contre la soldatesque française à la suite de la rumeur que l'Infant de la famille royale allait être enlevé, sur ordre de Murat. Les atrocités commises dépassent l'entendement, la populace, assoiffée de sang, s'est ruée sur les Français, qui ont répliqué par des gestes d'une cruauté inouïe et des massacres hallucinants.

3. Impossible de passer sous silence le livre d'Alessandro Baricco, Homère, Iliade (2006) où l'auteur n'a gardé que les passages qui font parler les hommes avant leur mort. S'insurgeant contre la boucherie devant Troie, Baricco termine sur une réflexion où se dessine une étincelle d'espoir devant le sang noirci : «Une réelle, prophétique et courageuse ambition pour la paix, je ne la vois que dans le travail patient et caché de millions d'artisans qui travaillent quotidiennement à faire naître une autre beauté, et la clarté de lumières, limpides, qui ne tuent pas » (p. 178). 